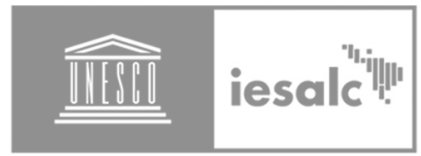

\title{
Unesco
}

Instituto Internacional

para la Educación Superior

en América Latina

y el Caribe
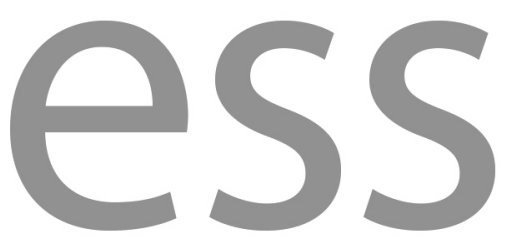

?

Educación

Superior y

Sociedad

Vol. 33 No. 2 (2021)

-

$\bullet$

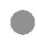

$\bullet$
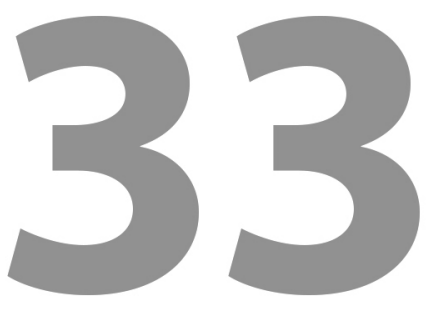

Incluye Dossier Temático:

Desafíos de la educación superior frente a la pandemia de Covid-19 en América Latina y el Caribe 


\section{Internacionalización de la educación superior en el oeste de Santa Catarina: la experiencia de una universidad comunitaria}

Silmara Terezinha Freitas, Maria de Lourdes Pinto de Almeida, Diego Palmeira Rodrigues, Karla Pezavento

\section{RESUMEN}

Este texto surge de un macroproyecto de investigación desarrollado por el Grupo Internacional de Estudios e Investigaciones de Educación Superior GIEPES UNICAMP, que investiga la internacionalización de la educación superior en territorio iberoamericano. En esta investigación participan veintidós (22) universidades de nueve (9) países, siendo una de ellas la Universidad del Oeste de Santa Catarina (Unoesc), institución caracterizada por el modelo comunitario, de carácter público de derecho privado, ubicado en el occidente de Santa Catarina. El objetivo de este artículo fue analizar cómo la Unoesc desarrolla sus procesos de movilidad académica en estudios de pregrado y posgrado, entre sus profesores, estudiantes y técnicos administrativos. La metodología científica utilizada en esta investigación fue la histórico-crítica, basada en la Pedagogía Histórico-Crítica, desarrollada por Saviani (2013), y que tiene como uno de los pilares maestros el materialismo histórico dialéctico. El instrumento de categorización fue el análisis de contenido de Bardin (2016). Como resultado, se evidenció que para la Unoesc la movilidad académica sigue siendo uno de los mayores frentes de internacionalización. Cabe mencionar que la internacionalización sigue siendo el camino para consolidar la investigación, la docencia y la extensión, con el objetivo de no perder su espacio frente al mercado competitivo. Además, cuenta con un organismo específico, desde 2010, para tratar temas relacionados con la movilidad académica, demostrando que desde hace 11 años esta Institución de Educación Superior se preocupa por tener frentes de internacionalización más allá del mercado capitalista.

Palabras clave: Internacionalización; Educación Superior; Universidad Comunitaria; Movilidad Académica. 


\title{
Internationalization of higher education in the west of Santa Catarina: the experience of a community university
}

\begin{abstract}
This text emerges from a macro research project developed by the International Group of Studies and Research of Higher Education - GIEPES UNICAMP, which investigates the internationalization of higher education in Ibero-American territory. In this research, 22 (twenty-two) universities from 9 (9) countries participate, one of them being the University of the West of Santa Catarina (Unoesc), an institution characterized in the community model, public of private law, located in western Santa Catarina. The aim of this article was to analyze how Unoesc develops its processes of academic mobility in undergraduate and graduate studies, among its professors, students and administrative technicians. The scientific methodology used in this investigation was the historical-critical, based on the Historical-Critical Pedagogy, developed by Saviani (2013), and which has as one of the master pillars the dialectical historical materialism. The categorization instrument was content analysis by Bardin (2016). As results, it was evidenced that for Unoesc academic mobility is still one of the largest fronts of internationalization. It is worth mentioning that internationalization is still the way to consolidate research, teaching and extension, aiming not to lose its space in the face of the competitive market. In addition, they have a specific body, since 2010, to deal with issues related to academic mobility, demonstrating that for 11 years this Institution of Higher Education is concerned with having internationalization fronts beyond the capitalist market.
\end{abstract}

Keywords: Internationalization; Higher Education; Community University; Academic Mobility.

\section{A internacionalização do ensino superior no oeste de Santa Catarina: a experiência de um colégio comunitário}

\section{RESUMO}

Este texto emerge de um projeto macro de pesquisa desenvolvido pelo Grupo Internacional de Estudos e Pesquisas de Educação Superior - GIEPES UNICAMP, que investiga a internacionalização da educação superior no território ibero- 
americano. Nesta investigação participam vinte e duas (22) universidades de nove (09) países, sendo uma delas, a Universidade do Oeste de Santa Catarina (Unoesc), instituição caracterizada no modelo comunitário, de natureza pública de direito privado, situada no oeste catarinense. $O$ objetivo deste artigo consistiu em analisar como a Unoesc desenvolve seus processos de mobilidade acadêmica na graduação e pós graduação, entre seus docentes, discentes e técnicos administrativos. A metodologia científica utilizada nesta investigação foi a históricocrítica, tendo como base a Pedagogia Histórico-Crítica, desenvolvida por Saviani (2013), e que tem como um dos pilares mestres o materialismo histórico dialético. $O$ instrumento de categorização foi análise de conteúdo de Bardin (2016). Como resultados, evidenciou-se que para a Unoesc a mobilidade acadêmica ainda é uma das maiores frentes de internacionalização. Vale a pena ressaltar que a internacionalização ainda é o caminho para a consolidação da pesquisa, do ensino e da extensão, visando não perder seu espaço frente ao mercado competitivo. Além disto, possui um órgão específico, desde 2010, para tratar dos assuntos voltados à mobilidade acadêmica, demonstrando que há 11 anos esta Instituição de Educação Superior está preocupada em ter frentes de internacionalização para além do mercado capitalista.

Palavras-chave: Internacionalização; Educação Superior; Universidade Comunitária; Mobilidade Acadêmica.

\section{Internationalisation de l'enseignement supérieur dans l'ouest de Santa Catarina: l'expérience d'une université communautaire}

\section{RESUMÉ}

Ce texte est le résultat d'un projet de macro-recherche développé par le Groupe international d'études et de recherche sur l'enseignement supérieur - GIEPES UNICAMP, qui étudie l'internationalisation de l'enseignement supérieur sur le territoire ibéro-américain. Vingt-deux (22) universités de neuf (9) pays participent à cette recherche, I'une d'entre elles est l'Université de l'Ouest de Santa Catarina (Unoesc), une institution caractérisée par le modèle communautaire, de caractère public de droit privé, située dans l'ouest de Santa Catarina. L'objectif de cet article était d'analyser comment l'Unoesc développe ses processus de mobilité académique dans les études de premier cycle et des cycles supérieurs, parmi 
ses professeurs, étudiants et techniciens administratifs. La méthodologie scientifique utilisée dans cette enquête était l'historico-critique, basée sur la pédagogie historico-critique, développée par Saviani (2013), et qui a comme l'un des piliers maîtres le matérialisme historique dialectique. L'instrument de catégorisation était l'analyse du contenu par Bardin (2016). Un résultat démontré c'est que pour l'Unoesc la mobilité académique reste l'un des plus grands fronts d'internationalisation. Il convient de mentionner que l'internationalisation est toujours le moyen de consolider la recherche, l'enseignement et l'extension, dans le but de ne pas perdre sa place face au marché concurrentiel. En outre, l'université dispose d'un organe spécifique, depuis 2010, pour traiter des questions liées à la mobilité académique, démontrant que depuis 11 ans, cet établissement d'enseignement supérieur se préoccupe d'avoir des fronts d'internationalisation audelà du marché capitaliste.

Mots clés: L'Internationalisation; L'Enseignement Supérieur; L'Université Communautaire; La Mobilité Académique.

\section{INTRODUCCIÓN}

Según Trevisol y Fávero (2019), el siglo XXI ha estado enmarcado por innumerables transformaciones en el sistema económico mundial, principalmente aquellas que interfieren en el modelo productivo y en el papel del Estado en relación a las políticas organizativas. Aun así, según los autores, resulta clara la tendencia hacia la comercialización de la educación superior, así como el contexto emergente para que las universidades se adapten a esta lógica.

En la contemporaneidad, las discusiones sobre internacionalización se han producido y convertido en un nuevo desafío para las universidades, por lo que se ven obligadas a adoptar determinadas políticas formuladas por organismos nacionales e internacionales, quienes también hacen las evaluaciones de las instituciones.

Para Miranda y Stallivieri (2017):

[...] o Brasil vive um momento em que a discussão sobre a necessidade da internacionalização da educação superior atinge um momento crucial, quando estão envolvidos os principais atores desse 
processo, como órgãos de governo, agências de fomento e entidades que representam a área da educação no país. Esse momento exige a identificação da existência, ou não, de fundamentos e definições para a construção de políticas públicas para a internacionalização do sistema de Ensino Superior(1) (p.592).

En este contexto, aparece el proyecto de investigación que sustenta este artículo. El objetivo consiste en analizar e identificar los procesos de internacionalización desarrollados en la Universidad del Oeste de Santa Catarina (Unoesc), en especial del Plan de Desarrollo Institucional (PDI) y del Plan Institucional de Internacionalización, así como los programas que desarrolla mostrando los impactos en la Universidad.

El trabajo presentado se basa en un Proyecto de Investigación Programa Institucional de Bolsas de Iniciação Científica del Conselho Nacional de Desenvolvimento Científico e Tecnológico (PIBIC/CNPQ)(2) sobre Internacionalización y Educación Superior, cuyo título principal es: "Internacionalización y educación superior: un estudio basado en la realidad encontrada en la Universidad del Oeste de Santa Catarina"; es parte de un macro proyecto del Grupo de Estudos e Pesquisas em Políticas de Educação Superior da Região Sul - GEPPES Sul - integrado a la red de la Universidade Estadual de Campinas (Unicamp).

El proyecto de matriz de la investigación fue elaborado por la Coordinadora del Grupo Internacional de Estudios e Investigación sobre Educación Superior (GIEPES) y socializado a grupos vinculados a esta red de investigación, y tiene como objetivo reflexionar sobre los procesos de internacionalización que se están desarrollando en las universidades, en este caso, en Unoesc.

(1) En español: “[...] Brasil vive un momento en el que la discusión sobre la necesidad de la internacionalización de la educación superior llega a un momento crucial, cuando se involucran los principales actores de este proceso, como agencias gubernamentales, agencias de desarrollo y entidades que representan el área educacional en el país. Este momento requiere la identificación de la existencia, o no, de fundamentos y definiciones para la construcción de políticas públicas para la internacionalización del sistema de Educación Superior".

(2) En español: Programa Institucional de Becas de Iniciación Científica del Consejo Nacional de Desarrollo Científico y Tecnológico. 
La metodología científica utilizada para desarrollar esta investigación fue histórico-crítica, utilizando como instrumento el análisis de contenido de Bardin (2013). En primer lugar, se buscó delimitar los caminos a seguir en la investigación, definiendo los procedimientos. Para ello, hemos hecho un inventario de las producciones académicas que trabajan este tema con el fin de ayudar en el desarrollo del marco teórico. Además, se verificó el perfil de los grupos de Educación de cada programa stricto sensu y las líneas de investigación que los componen.

A continuación, analizamos cómo aparecen las políticas de internacionalización en los documentos propios de la institución, como el PDI y el Plan de Internacionalización. Además del formulario de investigación de la Unicamp, en el que la universidad describió el desarrollo de acciones de movilidad académica, refiriéndose al envío y recepción de estudiantes, profesores y técnicos.

De esta forma, el producto del proyecto es el mapeo del futuro de la internacionalización en Unoesc. En este trabajo intentamos mostrar cómo Unoesc desarrolla sus procesos de movilidad académica. Se notan los impactos que ejercen los programas gubernamentales e institucionales en la universidad y como para Unoesc la internacionalización es el camino para la consolidación de la investigación, la enseñanza y la extensión, y de esta forma no perder su espacio ante al mercado competitivo y seguir las exigencias de las agencias evaluadoras.

\section{Sobre la investigación "Internacionalización de la educación superior en el territorio iberoamericano" (GIEPES-UNICAMP)}

El proyecto de investigación "Internacionalización y educación superior: un estudio basado en la realidad encontrada en la Universidad del Oeste de Santa Catarina", se desarrolla desde 2017 en Unoesc a través del Programa Institucional de Becas de Iniciación Científica (PIBIC), que busca apoyar la iniciación científica otorgando becas a estudiantes de pregrado. El Consejo Nacional de Desarrollo Científico y Tecnológico (CNPq), es un organismo del Ministerio de Ciencia, Tecnología, Innovaciones y Comunicaciones, cuyas principales funciones son promover la investigación científica y tecnológica y fomentar la formación de investigadores en Brasil, siendo, por tanto, el financiador de la investigación.

Con este proyecto se logró reflexionar sobre el proceso de internacionalización realizado por Unoesc, aportando una gran contribución sobre la producción académica y sus impactos en términos teóricos, políticos y prácticos para cada 
universidad comunitaria del estado de Santa Catarina. Además, el proyecto permitió un gran conocimiento de la internacionalización, además del intercambio y diálogo sobre las experiencias desarrolladas y en desarrollo en las universidades iberoamericanas.

La internacionalización ya es una realidad en las Instituciones de Educación Superior (IES) y frecuentemente se difunde el discurso de que la globalización ha cambiado el escenario de la búsqueda del conocimiento y que en este contexto existen demandas de adaptación de parte de las universidades a la internacionalización. En este sentido, el proyecto también tuvo como objetivo analizar los antecedentes que interfieren con estos discursos. Teniendo en cuenta que la Coordinación de Perfeccionamiento del Personal de Educación Superior (Capes), órgano del Ministerio de Educación, responsable del reconocimiento y evaluación de los posgrados stricto sensu a nivel nacional, ha exigido a los programas que desarrollen la internacionalización.

Considerando lo anterior, la internacionalización de la educación superior en Brasil se ha convertido en una de las mayores preocupaciones de las universidades y de algunas políticas adoptadas por organismos internacionales, como la Organización para la Cooperación y el Desarrollo Económico (OCDE), el Banco Mundial, las Naciones Unidas de Educación, Ciencia y Cultura (UNESCO) y de organismos nacionales (Capes, CNPq). En este campo de disputa, además de presuponer la interculturalidad, surge la integración de la investigación y el conocimiento, la solidaridad entre los pueblos y la aparición de otros actores e intereses que fomentan los debates y apuntan hacia la toma de decisiones relacionadas con políticas educativas (Trevisol y Fávero, 2019).

Partiendo de este contexto, para Silva (2017) la internacionalización ya no se considera un artículo de lujo, sino una cuestión de supervivencia para el escenario hodierno. Insertada en el mundo moderno caracterizado por la mercantilización, ha hecho que las universidades se hayan dado cuenta que internacionalizar significa buscar formas de competir con las mejores instituciones de educación superior a nivel nacional y mundial.

Otro aspecto que no se puede ignorar es que, si bien es un fenómeno importante en el contexto de las IES, la internacionalización presenta algunas inconsistencias. Y que, por tanto, no debemos ser ingenuos e irresponsables al considerar la internacionalización como algo estrictamente positivo pues, al fin y al cabo, ella implica una gran responsabilidad social y pedagógica. 


\section{La Universidad del Oeste de Santa Catarina (Unoesc): histórico e identidad}

La Universidad del Oeste de Santa Catarina (Unoesc) se define como una Institución Educativa Multicampi (Projeto de Universidade, 1991), cuya misión es ser un centro de referencia para el oeste de Santa Catarina, buscando la formación profesional y humana, a través de la docencia, la investigación y la extensión, generando conocimiento para el desarrollo regional sostenible (UNOESC, 2018). Aún en este contexto, la visión que tiene Unoesc, es ser reconocida y considerada la mejor institución de educación superior del oeste de Santa Catarina, destacando su relevancia social y de desarrollo, teniendo como valores la ética, el humanismo, el compromiso, la cooperación, la responsabilidad social y la solidez. Para reforzar esta idea citamos:

[...] A Unoesc nasceu e cresceu alicerçada nos seguintes valores: Ética: fundamentar as ações e os resultados na honestidade e na justiça; Humanismo: cuidado com a vida; Cooperação: capacidade de atuar em equipe; Comprometimento: atuar com profissionalismo para atingir objetivos; Responsabilidade social: desenvolvimento sustentável e qualidade de vida; Inovação: inovar com criatividade, flexibilidade e capacidade de adaptar-se a novas situações; e Solidez: garantir viabilidade presente e futura (UNOESC, 2018, p.18) ${ }^{(3)}$.

En 2019 ofreció más de cincuenta (50) cursos de pregrado en diferentes áreas, buscando el desarrollo humano, social, cultural, científico y tecnológico. Además de las especializaciones lato sensu y stricto sensu, la Universidad invierte en la mejora educativa de sus docentes y en la infraestructura, con laboratorios, aulas, canchas deportivas, equipamiento tecnológico, auditorios y un hospital.

\footnotetext{
${ }^{(3)}$ En español:"[...] Unoesc nació y creció a partir de los siguientes valores: Ética: basar acciones y resultados en la honestidad y la justicia; Humanismo: cuidado de la vida; Cooperación: capacidad para actuar en equipo; Compromiso: actuar con profesionalidad para alcanzar los objetivos; Responsabilidad social: desarrollo sostenible y calidad de vida; Innovación: innovar con creatividad, flexibilidad y capacidad de adaptación a nuevas situaciones; y Solidez: garantizar la viabilidad presente y futura" (UNOESC, 2018, p.18).
} 
Unoesc es mantenida por una fundación llamada Funoesc (Fundação do Oeste de Santa (atarina), que es una institución sin fines de lucro, y que también mantiene el Hospital Universitario Santa Terezinha (HUST) que sirve a la comunidad regional. Actualmente, la estructura de Funoesc se ubica desde el extremo oeste hasta el Vale do Rio do Peixe en once ciudades. Las unidades más grandes de la Universidad se encuentran en São Miguel do Oeste, Chapecó, Xanxerê, Videira y Joaçaba, donde se encuentra la sede legal de la fundación, la sede administrativa de la Universidad y el Hospital Universitario Santa Terezinha (UNOESC, 2018). El directorio y colegiado (formado por colaboradores universitarios, hospitalarios y comunitarios) coordinan la Funoesc y la Directoría es responsable de su administración, que incluye al Presidente y al Vicepresidente, Consejo Administrativo; Consejo Curador, y por la Asamblea General.

Sin embargo, para lograr la actual formulación, Unoesc ha sufrido varias transformaciones. Todo comenzó con la agregación de tres fundaciones educativas ubicadas en las ciudades polos de Joaçaba, Videira y Chapecó. La Fundação Educacional do Oeste Catarinense (FUOC), la Fundação Educativa e Empresarial do Alto Vale do Rio do Peixe (FEMARP) y la Fundação de Ensino para o Desenvolvimento do Oeste (FUNDESTE), resultando en la constitución de Unoesc (Ley Municipal $n^{\circ}$ 545 del de 28/11/68). En aquel momento, lo que se pretendía era la creación de una universidad que implementase la enseñanza, la investigación y la extensión.

La FUOC se transformó en Fundação Educacional Unificada do Oeste de Santa Catarina, permaneciendo como institución comunitaria, regional, con fines educacionales, regida por estatuto propio y por la legislación. La Unoesc permanece como entidad filantrópica, reconocida de Utilidad Pública Municipal (Ley ${ }^{\circ}$ 1.040/82), Estadual (Ley no 4.535/70) y Federal (Decreto no 87.741/82).

Con base en este parámetro, se puede destacar que, a partir de la década de 1970, la educación superior se instaló en el medio oeste de Santa Catarina, generando un avance significativo para la región. Sin embargo, es posible señalar que la región ya contaba con un potencial económico y social muy fuerte y estaba en pleno desarrollo (Projeto Universidade, 1991).

El oeste de Santa Catarina se caracteriza como una región dinámica, con un fuerte potencial económico y social y en pleno desarrollo. Hay sesenta y siete (67) municipios, que representan el $25 \%$ del área geográfica del estado, con una población de más de 1,5 millones de habitantes, lo que corresponde al 34\% de la población del estado (Projeto Universidade, 1991). 
Todavía en el periodo de origen de la Universidad, el occidente de Santa Catarina, área de alcance de la Unoesc, albergaba un vasto desarrollo económico en los tres sectores de la economía, con énfasis en los sectores secundario y terciario (Projeto Universidade, 1991). De esa manera, el modelo económico vigente en aquella época requería una institución que capacitara a profesionales para trabajar en campos emergentes. Así, Unoesc desarrolló su perfil, es decir, buscó ofrecer enseñanza, investigación, extensión y cursos de posgrado de calidad.

A partir de este parámetro, la construcción de una universidad en modelo multicampus se da por las exigencias del entorno, adaptándose a las necesidades de la sociedad de la época. Otro aspecto favorable a la creación de esta Universidad fue que las demás instituciones de educación superior se ubicaban en la costa, por lo tanto, lejanas para los residentes de las regiones del oeste de Santa Catarina. Eso significa que lo que se deseaba era "uma universidade inovadora, inserida em seu contexto histórico e geográfico e conhecedora profunda da realidade e dos problemas que envolvem ${ }^{(4) "}$ (Projeto Universidade, 1991, p. 18).

En esta coyuntura surge el debate de la internacionalización, haciéndose presente en la constitución de esta Universidad, desde el inicio de la segunda década de este siglo XXI, en principio como un desafío, tanto a nivel de licenciatura como de posgrado. Como señalan Trevisol y Fávero (2019), las universidades comunitarias de Santa Catarina y Rio Grande do Sul están bajo presión de ambos lados para internacionalizar sus agendas educativas (nacionales e internacionales), de una manera inmediata, recordando siempre que esta acción debe venir del ámbito local al global, y viceversa, sin perder el foco en su identidad.

De esa forma, no se puede retroceder en lo que atañe a la internacionalización, lo que se debe hacer es definir objetivos, fijar metas y monitorear los procesos para orientar los esfuerzos hacia una política institucional coherente.

\section{Políticas institucionales}

Según Stallivieri (2017), las universidades deben seguir un riguroso plan de internacionalización regido por metas y objetivos, cuya elaboración no es tarea fácil. Para la autora, un plan de inserción internacional efectivo resulta funda-

(4) En español: "una universidad innovadora, insertada en su contexto histórico y geográfico y con profundo conocimiento de la realidad y de los problemas que implica". 
mental para la elaboración de un diagnóstico sobre el perfil y misión de la institución.

En este contexto, Manjarrés y González (citado en Miranda y Stallivieri 2017) evidencian que " $(. .$.$) hay que aclarar algunos elementos fundamentales con el$ fin de ayudar en la elaboración de políticas de internacionalización" (p.592). Para ello, los autores proponen algunos parámetros, entre los que podemos mencionar identidad, institucionalidad, visibilidad, referencia y sostenibilidad. Con relación al parámetro de identidad hay que aclarar que se objetiva con la internacionalización y contribuye y responde a la construcción de un proyecto de país.

En cuanto al parámetro de institucionalidad, lo que se busca es la articulación de iniciativas y estrategias de diferentes actores y la ampliación de esfuerzos para diseñar conjuntamente una propuesta de internacionalización del sistema de educación superior, necesaria para el desarrollo del país. Para el parámetro de visibilidad se busca el reconocimiento de los avances y desafíos del país en la mejora de su imagen en el exterior, con el fin de dar visibilidad a las fortalezas de su sistema de educación superior y garantizar una oferta educativa de calidad.

Como parámetro de referencia, lo que destacan los autores Manjarrés y González (2014), es el desafío de transformar el sistema educativo a partir del reconocimiento de prácticas exitosas en otros países y nuestro propio país a través de IES y revisar los mecanismos internos para sistematizar, viabilizar y evaluar los avances de la internacionalización en el país. Y finalmente, el parámetro de sostenibilidad enfatiza la necesidad de continuar y ampliar el alcance de los programas e iniciativas que se están construyendo, para impulsar la internacionalización de la educación superior, asegurando su mantenimiento.

En la misma línea, Miranda y Stallivieri (2017) señalan que, para la construcción de una política pública de internacionalización en las universidades brasileñas, es necesario definir y aclarar los parámetros señalados por Manjarrés y Gonzáles (2014), que no se observan consistentemente en Brasil:

Identifica-se que há motivações econômicas, políticas, acadêmicas e socioculturais no suporte ao desenvolvimento econômico e tecnológico do país, carecendo ainda de indicações claras sobre as formas que a internacionalização da educação superior, a partir 
dessas motivações, contribuirá para o fortalecimento da posição internacional do Brasil no sistema mundial da educação. Somente a partir da construção de uma agenda positiva, do estabelecimento do diálogo constante e profícuo, da discussão e da elaboração de um documento nacional que envolva esses principais atores é que se irá produzir uma compreensão e uma visão de longo prazo sobre os objetivos estratégicos que a internacionalização da educação superior pode representar para o futuro e para a projeção do país em termos de inserção internacional. (Miranda y Stallivieri, 2017, p. 609)(5)

También según Gacel-Ávila y Rodríguez-Rodríguez (2018), son varios los obstáculos a los que se enfrenta una universidad para desarrollar un sólido programa de internacionalización. Entre ellos, los autores citan:

a) falta de estructuras institucionalizadas;

b) falta de planificación y ejecución;

c) gestión, mayoritariamente pasiva, con reproducción de documentos oficiales;

d) dificultades de comunicación en una lengua extranjera (desconocimiento);

e) financiación insuficiente para actividades internacionales.

En este contexto y como respuesta a nuestra propuesta de investigación, vamos a situar cómo se están llevando a cabo las políticas de internacionalización en la universidad comunitaria denominada Unoesc.

(5) En español: "Se identifica que existen motivaciones económicas, políticas, académicas y socioculturales en el soporte al desarrollo económico y tecnológico del país, careciendo aún de indicaciones claras sobre las formas con que la internacionalización de la educación superior, a partir de estas motivaciones, contribuirá al fortalecimiento del posicionamiento internacional de Brasil en el sistema educativo mundial. Solo construyendo una agenda positiva, estableciendo un diálogo constante y fructífero, discutiendo y elaborando un documento nacional que involucre a estos actores principales, se logrará una comprensión y visión de largo plazo de los objetivos estratégicos que puede representar la internacionalización de la educación superior al futuro y a la proyección del país en términos de inserción internacional". 
De acuerdo con su PDI, elaborado en 2018 y vigente hasta 2022, Unoesc afirma reconocer la importancia de impulsar y consolidar un proceso de internacionalización, considerándolo una necesidad del mundo contemporáneo, principalmente, los avances en la producción y socialización del conocimiento e innovaciones tecnológicas. En esta perspectiva los autores Gacel-Ávila y Rodríguez-Rodríguez (2018) afirman:

Declarar a la internacionalización como prioridad institucional requiere poner en marcha una serie de ajustes y reformas al quehacer y a las prácticas institucionales, tales como la integración de la dimensión internacional en los sistemas regulares de planeación, presupuestación y evaluación, la elaboración de planes operativos de internacionalización apegados a las prioridades institucionales, con identificación de los recursos financieros y humanos necesarios para asegurar su viabilidad, y el establecimiento de lineamientos y procedimientos de evaluación y monitoreo correspondientes (p. 125).

En cuanto a la política de internacionalización asumida por Unoesc, podemos destacar el deseo de desarrollar alianzas internacionales que aporten diferenciación y calidad en la formación de su comunidad académica para fortalecer el trípode existente de enseñanza, investigación, extensión, así como innovación. Además, la Universidad señala que mantendrá su Programa de Movilidad Académica, buscando insertar a la comunidad académica en el ámbito internacional, apuntando al desarrollo de las competencias y habilidades profesionales necesarias para el ejercicio profesional. Para lograr este propósito, la institución, según el PDI, estará ampliando la participación de estudiantes y profesores de pregrado y posgrado en eventos, intercambios, cursos, pasantías y redes de investigación y publicación, en conjunto con otras instituciones universitarias.

Al analizar el PDI, coincidimos con las ideas de Trevisol y Fávero, que la Unoesc al desarrollar acciones internacionalizadas se basa en los "[...] preceitos dos organismos internacionais que tratam do tema e dos documentos oficiais que regulam e formalizam o processo institucional da internacionalização(6) " (2019,

(6) En español: "preceptos de los organismos internacionales que tratan el tema y los documentos oficiales que regulan y formalizar el proceso institucional de internacionalización". 
p.12). Sobre todo, cuando se hace referencia a la internacionalización como una necesidad en el mundo globalizado y para competir en el mercado laboral.

Además, el Plan cuenta con unas directrices (aprobadas por la Res.01/CONSUN / 2018-21/02/2018), que son un principio orientador para las acciones a implementar. Entre las que podemos destacar:

a) implementar el proceso de internacionalización con la comunidad académica de la institución a través de programas y áreas de conocimiento;

b) asumir la internacionalización como un proceso integral y transversal a los procesos institucionales orientados a la enseñanza, la investigación, la extensión y la innovación, de manera inseparable;

c) ampliar y fortalecer la inserción de Unoesc en las redes internacionales de investigación;

d) posicionar a Unoesc en el espacio académico internacional.

Como análisis, es importante resaltar que Unoesc formaliza sus políticas de internacionalización de acuerdo con el discurso referente a la globalización, que trae en su núcleo los principios de innovación y tecnología.

Con base a este escenario, Unoesc también elaboró su primer Plan de Internacionalización (PI) en 2018. Este documento está dirigido exclusivamente a la internacionalización y tiene como objetivo fortalecer las acciones ya desarrolladas por la Universidad e implementar estrategias dirigidas al "incremento da produção e disseminação do conhecimento em benefício dos estudantes e profissionais envolvidos, tanto da Unoesc e quanto da comunidade regional como um todo(7) " (UNOESC-PI, 2018, p.4). Así presentamos el objetivo del PI de Unoesc, que según el propio documento es "apresentar uma proposta de planificação da internacionalização da Unoesc que permita a instituição organizar e priorizar suas ações, com objetivos, metas e recursos, visando à internacionalização do ensino superior e da pesquisa(8)" (UNOESC, 2018, p. 5).

\footnotetext{
(7) En español: "Incremento de la producción y difusión de conocimiento en beneficio de los estudiantes y profesionales involucrados, tanto de Unoesc como de la comunidad regional en su conjunto".

(8) “presentar una propuesta de planificación de la internacionalización de Unoesc que permita a la institución organizar y priorizar sus acciones, con objetivos, metas y recursos, visando a la internacionalización de la educación superior y la investigación".
} 
También se puede evidenciar que este Plan, tiene como objetivo reestructurar los procesos de internacionalización de la educación superior para trabajar con el trípode enseñanza-investigación-extensión, con miras a la integración y la cooperación, para alinear Unoesc con los tiempos contemporáneos.

Un aspecto destacado que se evidencia en el análisis tanto del PDI como del IP de Unoesc, es el Programa de Movilidad Académica. Este programa tiene como objetivo desarrollar competencias y habilidades académicas y profesionales en sus participantes. En este sentido, mencionamos que es común entre las universidades invertir en la movilidad académica como principal forma de internacionalización, debido al aporte de esta acción en la formación académica con la adaptación a otras culturas y el desarrollo de habilidades específicas en su área de conocimiento. Stallivieri (2009) explica que "[...] a formação em nível de graduação e pós-graduação com complementação no exterior, passou a ser muito valorizada em função do perfil profissional que está sendo solicitado pelo mercado $^{(9) "}$ (p.12). Según la autora, ésta es una de las principales justificaciones para promover la movilidad en las IES.

Sin embargo, señalamos la alerta de Knight (2012), cuando comenta que la movilidad académica se percibe erróneamente como la única vía de internacionalización, sin considerar las demás actividades que involucran este proceso. Además, frecuentemente favorece a los países desarrollados con el envío de académicos y no garantiza que sean bien recibidos por el país anfitrión.

Otro aspecto que se puede destacar en el PI (2018), es la visión de la universidad: "Ser reconhecida pela oferta de educação internacional de qualidade, com melhores oportunidades de internacionalização de currículo do oeste de Santa Catarina ${ }^{(10) "}$ (p.4). Lo que nos remite a Gough (citado en Thiesen, 2017, p. 1000), que alude que la internacionalización de los currículos puede entenderse como "creación de espacios transnacionales", donde las tradiciones del conocimiento local en la investigación curricular pueden ser protagonizadas y no como representaciones dentro de un discurso universal.

(9) En español: "la formación a nivel de pregrado y posgrado con complementación en el exterior, se ha vuelto muy valorada por el perfil profesional que está siendo solicitado por el mercado".

${ }^{(10)}$ En español:"Ser reconocidos por ofrecer educación internacional de calidad, con mejores oportunidades para la internacionalización curricular en el oeste de Santa Catarina". 
Para ello, es fundamental que la universidad desarrolle un plan orientado a su realidad y que se coloque como protagonista, lo que no es tarea fácil ya que, como señalan Manjarrés y Gonzáles (2014), todavía no hay un camino marcado para Brasil y que también está siguiendo los discursos promovidos.

Además, también cabe mencionar a la autora Betty Leask (citado en Thiesen, 2017, p.1003), al ponderar la importancia de reflexionar sobre dos conceptos similares, pero que presentan algunas distinciones: "el concepto de currículo internacionalizado e internacionalización del currículo".

La internacionalización del currículo sería la posibilidad de incorporar al contenido a la enseñanza, al aprendizaje y a los instrumentos de evaluación, las dimensiones interculturales e internacionales. El plan de estudios internacionalizado es aquel que involucrará a estudiantes en la investigación internacional y diversidad lingüística y cultural, además de desarrollar sus perspectivas interculturales e internacionales como ciudadanos y profesionales globales.

En cuanto a los objetivos generales y específicos, Unoesc busca promover el desarrollo académico y la difusión del conocimiento a nivel internacional, necesitando reestructurar el proceso de internacionalización e investigación. Para lograr este macroobjetivo, la Universidad apunta a los siguientes objetivos:

1) desenvolver competências interculturais e o plurilinguismo no ambiente universitário, incentivando a internacionalização de currículo e a internacionalização doméstica;

2) incrementar a cooperação internacional e a captação de recursos para o desenvolvimento conjunto de atividades técnico-científicas e de programas internacionais;

3) aumentar o número de alunos e docentes em intercâmbio internacional, em níveis de graduação e pós-graduação, integrado ao processo de ensino-aprendizagem;

4) organizar o processo de dupla titulação e de cotutela no âmbito da pós-graduação; 
5) incentivar a publicação internacional qualificada dos docentes pesquisadores;

6) institucionalizar a internacionalização e seus respectivos processos de gestão no âmbito do ensino superior(11). (UNOESC, 2018, p.5)

Es fundamental evidenciar que este es el primer documento formulado por la Universidad con una perspectiva exclusiva a la internacionalización y que se vienen desarrollando diversas acciones para estructurar los procesos. Entre estas acciones, podemos mencionar el Programa de Movilidad Académica, la oferta de lenguas extranjeras; convenios bilaterales con universidades; el fortalecimiento de la diplomacia cultural entre países; envío periódico de profesores y estudiantes para participar en intercambios, investigaciones y congresos; inversión en formación docente e incentivo a la formación de redes de investigación; y dar la bienvenida a estudiantes, profesores e investigadores que realicen intercambios culturales, académicos, tecnológicos y científicos.

\section{2 .ANÁLISIS DE UN INSTRUMENTO DE COLECTA DE DATOS}

De acuerdo con los parámetros de la Unoesc, para colaborar con el desarrollo de la internacionalización, la institución busca implementar proyectos que permitan su avance. Uno de ellos es el Proyecto de Movilidad Académica creado en 2010 como una de las primeras acciones de internacionalización de la Universidad, que, con este Programa y la institución de un coordinador específico para planificar y ejecutar acciones estratégicas, asumió el compromiso con el desarrollo y la formación humana de estudiantes y profesores.

(11) En español:"1) desarrollar las competencias interculturales y el plurilingüismo en el ámbito universitario, fomentando la internacionalización curricular y la internacionalización nacional; 2) incrementar la cooperación internacional y la captación de fondos para el desarrollo conjunto de actividades técnico-científicas y programas internacionales; 3 ) incrementar el número de estudiantes y docentes en intercambio internacional, a nivel de pregrado y posgrado, integrados en el proceso de enseñanza-aprendizaje; 4) organizar el proceso de doble titulación y cotutela en el ámbito de los estudios de posgrado; 5) fomentar la publicación internacional calificada de investigadores docentes; 6 ) institucionalizar la internacionalización y sus respectivos procesos de gestión en el contexto de la educación superior (UNOESC, 2018, p.5). 
Otro aspecto relevante del Proyecto de Movilidad Académica se refiere al desarrollo de las competencias y habilidades profesionales necesarias para el ejercicio profesional y también de los conocimientos, valores y actitudes científicotécnicas para el ejercicio de la ciudadanía en Brasil y en el exterior. Abarca a estudiantes de grado y posgrado de la IES, brindando a profesores y estudiantes investigaciones científicas y prácticas curriculares obligatorias y no obligatorias, además de cursos de extensión y congresos, con una duración de un semestre a un año académico.

Según una entrevista realizada en 2017 con la coordinadora del Programa, Kaline Zeni, Unoesc tiene acuerdos en diferentes continentes, como Norteamérica, Latinoamérica, Europa y África. Sin embargo, estos acuerdos están en constante evolución para que se firmen nuevas alianzas. Estos convenios están a cargo de la Rectoría y Coordinación General de Movilidad Académica, así como a través de propuestas de términos de cooperación que presenten los profesores permanentes de los posgrados stricto sensu de la institución.

Además de la movilidad académica, Unoesc se incorporó al Programa Ciencia sin Fronteras, del Ministerio de Ciencia, Tecnología e Innovación, brindando oportunidades para el intercambio de estudiantes tanto en la modalidad de cuotas de la IES como de solicitudes individuales. Otro programa de la Universidad es el Programa Estudiantes Convenio de Pregrado - del Ministerio de Educación (MEC).

Desde 2010 la coordinadora del sector de Movilidad Académica es la profesora Kaline Zeni, siendo responsable de la coordinación general y también del Campus de Joaçaba, actuando junto con la secretaria Glaucia de Césaro Luthi. Cabe destacar que en cada campus hay un responsable del sector que se reporta a Kaline como coordinador. Los responsables no tienen este cargo como exclusividad, por ello, desarrollan otras funciones dentro de la Universidad y actúan también como representantes locales de la movilidad académica.

Otro punto a destacar de la entrevista concedida por la profesora Kaline Zeni en 2017, se refiere a los convenios firmados por la institución, que abarcan todas las áreas del conocimiento, y que, por tanto, dependen de la gestión del uso, es decir, del interés. De modo que la Universidad busca firmar pocos convenios, pero de calidad; que sean efectivos. Si un contrato en particular no satisface las necesidades e intereses académicos, la Universidad buscará otros acuerdos.

Volviendo al objetivo del presente estudio, como instrumento de investigación para el mapeo y análisis de los procesos de internacionalización de Unoesc, se 
utilizaron los formularios desarrollados por el macro proyecto del GIEPES - UNICAMP, los cuales fueron aplicados a veintidós (22) universidades de los siguientes países: Argentina, Brasil, Colombia, Chile, España, México, Portugal, Uruguay y Venezuela.

Una de las universidades brasileñas participantes en el proyecto GIEPES fue Unoesc y en vista de lo anterior, pudimos elaborar la siguiente tabla con los datos ofrecidos por la propia Universidad, de las acciones de internacionalización desarrolladas por la institución en el periodo 2013-2017:

Tabla 1. Datos cuantitativos de las acciones de movilidad de Unoesc entre 2013 y 2017

\begin{tabular}{|c|c|}
\hline DESCRIPCIÓN & CANTIDAD \\
\hline Número de alumnos de posgrado con Beca Sándwich en el exterior & 01 por año desde 2014 \\
\hline Número de alumnos con beca Sándwich del exterior en la IES & 01 \\
\hline Número de alumnos de la institución en el exterior & 101 \\
\hline Número de alumnos del exterior en la institución & 28 \\
\hline $\begin{array}{l}\text { Número de alumnos recibidos por medio del Programa } \\
\text { de Estudiantes-Convenio de Graduación (PECG) }\end{array}$ & 13 \\
\hline Número de profesores visitantes & 11 \\
\hline Número de docentes da instituição em pós-doutorado no exterior & 29 \\
\hline Número de proyectos actualmente en desarrollo & 25 \\
\hline Número de eventos con participación internacional & 23 \\
\hline
\end{tabular}

Fuente: Elaboración propia.

A la vista de los datos presentados en esta tabla, es evidente que Unoesc viene desarrollando acciones en materia de internacionalización, una gran parte de ellas actividades orientadas a la movilidad académica. Sin embargo, Santos y Filho (2012) advierten que existen varias formas de internacionalización, como la movilidad; la escala y ambición en la investigación científica; la internacionalización de las actividades de transferencia e innovación; evaluación de buenas prácticas y diplomacia cultural universitaria. 
Otro aspecto que llama la atención está en el envío y recepción de estudiantes y docentes. Resulta evidente que el envío de alumnos y profesores es mayor que el número de recibidos. Por ejemplo, al comparar la cantidad de estudiantes con becas sándwich que se han recibido, tenemos sólo uno (1) y desde 2014, se ha enviado al menos un (1) estudiante. Otro dato es en relación al número de estudiantes del exterior en la Universidad, con veintiocho (28) en total, y los enviados corresponden a ciento uno (101).

Esto nos remite a lo que informa Leal (2018) sobre la internacionalización en beneficio de países que tienen un mayor desarrollo económico y tecnológico en detrimento de los demás países, siguiendo la lógica del capitalismo. Para la autora, el origen colonial de la internacionalización refuerza geografías desiguales de poder, conocimiento y ser. Ese escenario se basa en la desigualdad y la jerarquía, legitimando ciertos países, universidades, individuos, conocimientos y modos de existencia como naturalmente superiores a otros.

Retomando los datos de las acciones de internacionalización desarrolladas por la institución, en relación a los programas gubernamentales de promoción de la internacionalización adheridos por Unoesc, se destaca el Programa de Estudiantes-Convenio de Graduación (PEC-G) a través del cual la Universidad recibe académicos extranjeros casi todos los años. Este Programa existe desde hace más de 50 años y es una actividad de cooperación internacional, cuyo objetivo es la formación de recursos humanos, con el fin de capacitar a ciudadanos de países en desarrollo (África, América Latina y el Caribe) con los que Brasil tiene convenios educativos o culturales, para cursar estudios universitarios en el país, a nivel de pregrado, en IES brasileñas participantes en el Programa.

El PEC-G es administrado por el Ministerio de las Relaciones Exteriores por medio de la División de Cooperación Educacional del Departamento de Cooperación Científica Técnica y Tecnológica (DCE/DCT/MRE) y por el Ministerio de la Educación, por medio de la División de Asuntos Internacionales de la Secretaría de Educación Superior (DAI/ SESu/MEC).

Los interesados en participar en el Programa deben comunicarse con las embajadas u oficinas consulares del país de origen para su registro. Unoesc anualmente ofrece nuevos cursos y plazas al Programa PEC-G y ha recibido a varios estudiantes de Paraguay, Cabo Verde, Ecuador, Guinea-Bissau y Angola. Lo que llama la atención es que el estudiante extranjero que esté dispuesto a estudiar en una institución brasileña, debe asumir todos los costos y esto debe acreditarse mediante una declaración de condiciones económicas. 
Otro dato relevante de Unoesc está en relación al número de proyectos desarrollados en alianzas con universidades extranjeras, siendo un total de veinticinco (25), los cuales suelen ser desarrollados por las redes de investigación y coordinados por los profesores de los programas stricto sensu de la Universidad.

En este sentido, cabe mencionar que las redes de investigación son una forma de internacionalización en la que los involucrados no necesitan viajar a países, sino construir conocimiento a través de la colaboración y la producción. Según Leite (2014)"uma rede se estabelece quando um grupo de pessoas, instituições, agências, empresas, estão em contato [...]" (p.310)(12). Una red de investigación y colaboración tiene los mismos atributos definidores y agrega a ellos la intención de producir conocimiento.

\section{CONSIDERACIONES FINALES}

Hoy en día, es evidente que la movilidad académica se está fundamentando como una estrategia de internacionalización. Este hecho se contribuye para su promoción global, principalmente en relación a los países más desarrollados, que en consecuencia presentan mayor capacidad tecnológica y condiciones de recibir a los estudiantes de intercambio.

Con el incremento de la competencia provocado por el avance de la economía y los procesos productivos, tanto en términos científicos como tecnológicos, la internacionalización significa buscar formas de competir con las mejores instituciones de educación superior, tanto a nivel nacional como global. En este contexto, la internacionalización de la educación no simboliza la opulencia, sino una cuestión de supervivencia para las universidades contemporáneas.

Por otro lado, Lee (2013) advierte que es ingenuo e irresponsable ver la internacionalización como algo necesariamente positivo, y que debemos reflexionar sobre estos procesos. Un ejemplo sería el informe de algunos estudiantes de intercambio que se quejan de sufrir discriminación y que vendría del alumnado y profesorado del país anfitrión.

Lo que se desea resaltar es que Unoesc viene desarrollando la internacionalización en cursos de grado y posgrado, especialmente en lo que se refiere al

(12) En español: "una red se establece cuando un grupo de personas, instituciones, agencias, empresas, están en contacto [...]. 
tema de la movilidad académica de docentes y estudiantes. Lo mismo ocurre en los programas stricto sensu, a través de convenios, que se enfocan en la investigación y la producción.

La investigación también nos mostró que el Programa de Movilidad Académica, frecuentemente, tiene como objetivo desarrollar las competencias y habilidades profesionales necesarias para el ejercicio de la carrera, además de traer un bagaje cultural inconmensurable a los involucrados en el proceso pedagógico. Y que las iniciativas institucionales, cuando no son el resultado de programas o proyectos impulsados por agencias gubernamentales, demuestran la voluntad política institucional para desarrollar determinadas acciones, siendo esta una de las situaciones en las que la autonomía de la universidad puede ser plenamente efectivada.

En este contexto, estamos de acuerdo con las ideas de Trevisol y Fávero (2019) cuando dicen que es necesario evidenciar que las políticas de internacionalización en las instituciones requieren "comprometimiento y planeamiento".

Finalmente, se puede concluir que la movilidad académica es el primer gran camino para la internacionalización de las universidades, especialmente cuando se entiende como una necesidad de la sociedad del conocimiento. Y que Unoesc, como institución multicampus y comunitaria, está asumiendo su compromiso con la región de actuación y que con los esfuerzos que presenta, sugiere la solidificación de la internacionalización en los próximos años.

\section{REFERENCIAS}

Bardin, Laurence (2016). Análise de conteúdo. Edições 70.

Decreto Federal n 87.741, de 25 de outubro de 1982 (1982). Declara de utilidade pública as instituições que menciona. Diário Oficial da União, Seção 1 de 26/10/1982. Brasília, DF, Brasil. Presidência da República do Brasil.

Gacel-Ávila, J. y Rodriguez-Rodriguez, S. (2018). Internacionalización de la educación superior en América Latina y el Caribe: un balance. UNESCO-IESALC.

Knight, J. (2012). Cinco verdades sobre a internacionalização. Revista Ensino Superior - UNICAMP. https://www.revistaensinosuperior.gr.unicamp.br/international-highereducation/cinco-verdades-a-respeito-da-internacionalizacao 
Leal, F. G. (2018). Internacionalização invertida: reflexões críticas sobre a educação superior mundial contemporânea. https://iberoamericasocial.com/internacionalizacaoinvertida-reflexoes-criticas-sobre-a-educacao-superior-mundial-contemporanea/

Lee, J. J. (2013). O falso glamour da internacionalização das universidades. Revista Ensino Superior UNICAMP. https://www.revistaensinosuperior.gr.unicamp.br/international-higher-education/o-falso-glamour-da-internacionalizacao-das-universidades

Lei Estadual n 4535, de 15 de outubro de 1970. (1970). Declara de utilidade pública a Fundação Universidade do Oeste de Santa Catarina (FUNOESC), de Joaçaba. Diário Oficial 9.110 de 22/10/70. Florianópolis, SC, Brasil. Governo do Estado de Santa Catarina.

Lei Municipal n. 545, de 22 de novembro de 1968. (1968). Institui a Fundação Universitária do Oeste Catarinense (FUOC). Diário Oficial. Joaçaba, SC, Brasil. Prefeitura Municipal de Joaçaba.

Lei Municipal no 1.040, de 14 de maio de 1982. (1982). Declara de Utilidade Pública Municipal a Fundação Universidade do Oeste de Santa Catarina (FUNOESC). Diário Oficial. Joaçaba, SC, Brasil. Prefeitura Municipal de Joaçaba.

Leite, Denise; Caregnato, Célia Elizabete; Lima, Elizeth Gonzaga dos Santos; Pinho, Isabel; Miorando, Bernardo Sfredo y Silveira, Priscila Bier da. (2014). Avaliação de redes de pesquisa e colaboração. Avaliação (Campinas).Vol.19, n.1, pp.291-312. ISSN 1414-4077. http://dx.doi.org/10.1590/S141440772014000100014

Manjarrés, N. J. y González, C. A. (2014). Elementos para la construcción de una política pública de internacionalización de la educación superior. En: Salmi, J. et al. (Orgs.) Reflexiones para la política de internacionalización de la educación superior en Colombia. Ministerio de Educación Nacional. pp. 267-283.

Miranda, José Alberto Antunes de y Stallivieri, Luciane (2017). Para uma política pública de internacionalização para o ensino superior no Brasil. Avaliação, Campinas; Sorocaba, SP, v. 22, n. 03, pp. 589-613.

Santos, F. S. y Filho, N. de Almeida (2012). Internacionalização universitária na sociedade do conhecimento. Universidade de Brasília. Imprensa da Universidade de Coimbra. 
Saviani, Dermeval (2013). Pedagogia histórico-crítica: primeiras aproximações. 11 ed. Autores Associados.

Silva, L. L. (2017). Políticas de formação de professores (as) universitários (as) em São Paulo e Catalunha: tendências e desafios. Educação e pesquisa, v. 43, n. 1, pp. 113-126. https://doi.org/10.1590/s1517-9702201701158662

Stallivieri, Luciana (2009). As dinâmicas de uma nova linguagem intercultural na mobilidade acadêmica internacional. Tese (Doutorado em Línguas Modernas) - Universidad Del Salvador, Buenos Aires, 2009.

Stallivieri, L. (2017). Internacionalização e intercâmbio: dimensões e perspectivas. Appris.

Thiesen, Juares da Silva. (2017) Internacionalização dos currículos na educação básica: concepções e contextos. Revista e-Curriculum, São Paulo, v. 15, n. 4, p. 991-1017, out./dez.

Trevisol, M. G.; Fávero, A. (2019). As diversas faces da internacionalização: análise comparativa entre duas instituições comunitárias do sul do Brasil. Revista Internacional de Educação Superior, Campinas, SP, v. 5, p. 1-22. https://doi.org/10.20396/riesup.v5i0.8653894

Universidade do Oeste de Santa Catarina (1991). Projeto de universidade. Fundação Educacional Unificada do Oeste de Santa Catarina. Joaçaba/Chapecó/Videira. Evangraf.

UNOESC (2018). Universidade do Oeste de Santa Catarina. Plano de Desenvolvimento Institucional - PDI (2018-2022). 3. ed. Ed. Unoesc. https://www.unoesc.edu.br/images/uploads/atendimento/PDI___web_2019.pdf 


\section{BIONOTAS}

Silmara Terezinha Freitas. Doutoranda em Educação na Universidade do Oeste de Santa Catarina (UNOESC), Brasil. Mestre em Educação (2019). Graduada em Matemática (2011) e Física (2006). Pesquisadora do Grupo de Estudos e Pesquisas em Políticas de Avaliação e Accountability em Países da América Latina (GEPAyA).

Correo electrónico: silmara.fisica@gmail.com https://orcid.org/0000-0003-1657-4781

Maria de Lourdes Pinto de Almeida. Historiadora e Pedagoga. Mestre e Doutora em História, Filosofia e Educação pela FE da Unicamp. Pós-doutorado em Políticas Educacionais pela Unicamp. Docente pesquisadora do PPGEd da Unoesc. Editora da RIESup-Unicamp. Coordenadora Adjunta do GIEPES-Unicamp, da RIEPPES-Unoesc/Unicamp e do GEPPES SUL-Unoesc/Unicamp.

Correo electrónico: malu04@gmail.com

https://orcid.org/0000-0001-8515-2908

Diego Palmeira Rodrigues. Graduado em Educação Física pela UFSM, Mestre e Doutorando em Educação pela UNOESC com bolsa UNIEDU-FUMDES, pesquisando Políticas e Processos de Internacionalização. Técnico em Assuntos Educacionais da UFFS. Pesquisador da RIEPPES-UNOESC/UNICAMP e do GIEPES-UNICAMP. Editor de seção da Revista RIESup-UNICAMP.

Correo electrónico: diegopalmeirarodrigues@gmail.com https://orcid.org/0000-0002-2431-654X

Karla Pezavento. Possui graduação em Pedagogia pela UNOESC. Estudante pertencente ao Grupo de Estudos GEPPES SUL-UNICAMP e integrante da RIEPPES. Secretária Geral e Executiva da Revista RIESup. Mestranda em Educação do PPGEd UNOESC, investigando Políticas e Processos de Internacionalização.

Correo electrónico: kapezavento@gmail.com https://orcid.org/0000-0002-2065-2239 